\title{
Expressions of mRNA for innate immunity-associated functional molecules in urinary sediment in immunoglobulin A nephropathy
}

\author{
KAZUSHI TSURUGA, ${ }^{1}$ TOMOMI AIZAWA, ${ }^{1}$ SHOJIRO WATANABE, ${ }^{1}$ KOJI TSUGAWA, ${ }^{1}$ HIDEMI YOSHIDA, ${ }^{2}$ \\ TADAATSU IMAIZUMI, ${ }^{2}$ ETSURO ITO ${ }^{1}$ and HIROSHI TANAKA ${ }^{1,3}$ \\ ${ }^{1}$ Department of Pediatrics, Hirosaki University Hospital, ${ }^{2}$ Department of Vascular Biology, Graduate School of Medicine and ${ }^{3}$ Department of School Health \\ Science, Faculty of Education, Hirosaki University, Hirosaki, Japan
}

\section{KEY WORDS:}

childhood IgA nephropathy, fractalkine, innate immunity, non-invasive method, urinary sediment.

\section{Correspondence:}

Dr Hiroshi Tanaka, Department of Pediatrics, Hirosaki University Hospital, Hirosaki 036-8563, Japan. Email: hirotana@hirosaki-u.ac.jp

Accepted for publication 3 June 2015. Accepted manuscript online 7 June 2015.

\section{doi:10.1111/nep.12533}

Conflict of interest: On behalf of all authors, the corresponding author states that there is no conflict of interest.

\section{SUMMARY AT A GLANCE}

In this article, the authors measured the urinary mRNA levels of several innate immunity associated functional molecules in paediatric IgAN patients and demonstrated that fractalkine was significantly correlated with the histological activity index and the chronicity index. They concluded that measurement of mRNA expression of fractalkine in urinary sediment could use as a non-invasive method for predicting histologic severity in IgAN in children.

\begin{abstract}
:
Aim: It has been reported that the innate immune system plays a pivotal role in the pathogenesis of immunoglobulin A nephropathy (IgAN). To explore non-invasive monitoring of disease activity in children with IgAN, we examined whether expressions of mRNA for innate immunityassociated functional molecules: CC ligand chemokine 5 (CCL5), fractalkine/ CX3CL1, interferon- $\gamma$-induced protein 10 (IP-10), monocyte chemoattractant protein 1 (MCP-1), retinoic acid-inducible gene-I (RIG-I), and toll-like receptor 3 (TLR3) in urinary sediment from patients with IgAN correlate with histologic parameters.

Methods: Twenty consecutive children with IgAN and four children with thin basement membrane disease (serving as a non-inflammatory control) were enrolled in this pilot study. Urinary mRNA expressions of target genes were examined real-time quantitative polymerase chain reaction.

Results: The expressions of CCL5, fractalkine and RIG-I were significantly increased in IgAN (all $P<0.05$ ). Although no significant correlation was observed between mRNA expressions of these three molecules and clinical parameters, such as levels of urinary protein excretion, degrees of occult blood in urine and serum albumin, the expression of fractalkine was significantly correlated with the histological activity index $(P=0.022)$ and the chronicity index $(P=\mathbf{0 . 0 0 5})$. Furthermore, intense glomerular immune activity of fractalkine was observed in biopsy specimens in patients with moderately to severe proliferative IgAN.

Conclusion: Regional expression of fractalkine may be involved in the pathogenesis of childhood IgAN. Although our present findings remain preliminary, measurement of mRNA expression of fractalkine in urinary sediment could be used as a non-invasive method for predicting histologic severity in IgAN in children. Further studies of this issue are needed.
\end{abstract}

Since microbial infections trigger the worsening and sometimes the onset of immunoglobulin A nephropathy (IgAN), the innate immune system is thought to play a pivotal role in the pathogenesis of the disease. ${ }^{1-7}$ The activation of toll-like receptors (TLRs) and their downstream immune responses can be induced by both infectious pathogens and noninfectious stimuli such as endogenous ligands, and this mechanism may be involved in the pathogenesis of immunocomplex-mediated glomerulonephritis, including
IgAN. ${ }^{8}$ In experimental and clinical settings, it has been reported that regional expressions of proinflammatory cytokines/chemokines in glomerular cells can be induced by the activations of TLR3 and/or TLR4 and their downstream signalling pathways. ${ }^{1-9}$ Moreover, in a recent interesting experiment, viral dsRNA suppresses human podocyte cell function via the activations of TLR3 and retinoic acid inducible gene-I (RIG-I), a putative RNA helicase acting as a cytoplasmic sensor of RNA viruses. ${ }^{7}$ Thus, innate immunity 
against infectious pathogens in residual glomerular cells may be involved in the pathogenesis of IgAN.$^{1-7}$

It has recently been reported that quantification of messenger RNA expression of certain target genes in urinary sediment by reverse transcription became possible, and realtime quantitative polymerase chain reaction (RT-qPCR) may be a valuable noninvasive method for studying kidney diseases. ${ }^{10-14}$ We previously examined mRNA expression of transcriptional factors for the differentiation of helper $\mathrm{T}$ lymphocytes toward Th1, T-bet and Th2, GATA-3 in urinary sediment of children with IgAN and purpura nephritis (PN), and found that newly diagnosed cases showed a higher expression of T-bet in urinary sediment compared to controls, and that this higher expression was associated with positive staining of T-bet protein in renal biopsied tissue. ${ }^{12,13}$ In an experimental setting, we also examined the TLR3 signalling cascades treated with polyinosinic-polycytidylic acid (poly IC), a synthetic analogue of viral dsRNA, that makes 'pseudoviral' infection in cultured human mesangial cells (MCs), and found that the activation of mesangial TLR3 upregulated the expressions of monocyte/macrophage and lymphocyte chemoattractants: CC ligand chemokine 5 (CCL5), fractalkine /CX3CL1, interferon (IFN)- $\gamma$-induced protein 10 (IP-10), and monocyte chemoattractant protein-1 (MCP-1), in cultured human MCs. ${ }^{1,4,5,8,9}$ In this paper, to explore non-invasive monitoring of disease activity in childhood IgAN, we therefore examined whether the expressions of mRNA for CCL5, fractalkine, IP-10, MCP-1, RIG-I and TLR3 in urinary sediment from patients with IgAN correlate with histologic severity.

\section{METHODS}

This study was approved by the ethics committee of Hirosaki University Graduate School of Medicine. Urine samples were collected after obtaining written informed consent from the patients' parents.

Twenty consecutive paediatric patients who were newly diagnosed with IgAN and four children with thin basement membrane disease (TBMD, a non-inflammatory kidney disease serving as a non-inflammatory control) at our hospital were enrolled in this pilot study. In this study, we used TBMD as a control, because such patients had glomerular haematuria due to biopsy-proven, noninflammatory mechanism. The IgAN patients were 10 boys and 10 girls with a median age of 10.3 years $(10.3 \pm 4.8$ years $)$ at entry, whereas the TBMD patients were three boys and a girl with a median age of 13.0 years ( $13.0 \pm 3.6$ years). No patient received drug therapy at entry, since all patients were detected during a mass screening system for school children in Japan. All the patients had a significant haematuria, but normal blood pressure and normal renal function at presentation. No patient had active infection at that time. The mean values for urinary protein/creatinine ratio (Up/cr) in the study participants were $1.9 \pm 2.4$ in IgAN patients and $0.1 \pm 0.1$ in TBMD patients, respectively. A diagnostic renal biopsy was performed within 3 months from the initial detection of urinary abnormalities in all of them. The biopsy specimens were scored semiquantitatively, using the scoring system for childhood IgAN described by Andreoli and Bergstein. ${ }^{15}$ The activity index (AI) was determined by grading mesangial proliferation, interstitial mononuclear cell infiltration, and cellular crescent formation using a 4-grade scale ( 0 , none; 1 , mild; 2 , moderate; 3 , severe), based on the percentage of glomeruli involved $(0,0 \% ; 1,1-20 \% ; 2,21-50 \% ; 3$, $>50 \%$ ). The chronicity index (CI) was determined by counting the number of glomeruli demonstrating fibrous crescents and segmental or global sclerosis, each being scored on a scale of $0-3$, on the basis of the percentage of glomeruli involved as indicated. Tubular atrophy and interstitial fibrosis were each scored on a scale of 0-3. The sum of these numbers comprised the activity index $($ maximum $=9)$, and the chronicity index $($ maximum $=12)$. In the IgAN patients, $\mathrm{AI}$ and $\mathrm{CI}$ were $3.4 \pm 1.5$ and $3.1 \pm 1.2$, respectively.

A whole-stream, early morning, urine sample at the time of renal biopsy was collected for the gene expression study. ${ }^{11-13}$ Briefly, the urine samples were centrifuged at $1650 \mathrm{~g}$ for $30 \mathrm{~min}$. Total RNA was extracted using an RNeasy Mini Kit (Qiagen Science, Germantown, $\mathrm{MD}$, USA), according to the manufacturer's instructions. All the specimens were stored at $-80^{\circ} \mathrm{C}$. The purity of RNA was confirmed by the relative absorbance at a 260/280 $\mathrm{nm}$ ratio. We quantified the mRNA expression of CCL5, fractalkine, IP-10, MCP-1, RIG-I and TLR3 in the urinary sediment. For each reaction, approximately $0.1 \mu \mathrm{g}$ of total RNA was reverse transcribed to cDNA using an iScript cDNA synthesis Kit (Bio-Rad Laboratories, Hercules, CA, USA). The resulting CDNAs were stored at $-80^{\circ} \mathrm{C}$ until required. The relative mRNA abundance was quantified using CXF96 Touch (Bio-Rad Laboratories). The mRNA expression level of each target was normalized to that of the housekeeping gene, 18S rRNA. PCR amplifications were performed in $20 \mu \mathrm{L}$ at $95^{\circ} \mathrm{C}$ for $3 \mathrm{~min}$, followed by 40 cycles of incubation at $95^{\circ} \mathrm{C}$ for $10 \mathrm{~s}$ and at $60^{\circ} \mathrm{C}$ for $30 \mathrm{~s}$. Each sample was run in triplicate. The results were analyzed with a CFX Manager (Bio-Rad Laboratories). To quantify the abundance of the target mRNAs, we calculated the differences in the threshold cycles between the target genes and $18 \mathrm{~S}$ rRNA. The primer sequences were as follows: CCL5: -forward, 5'- CTA CTC GGG AGG CTA AGG CAG GAA - $3^{\prime}$, reverse, 5'- GAG GGG TTG AGA CGG CGG AAG C - $3^{\prime}$-; fractalkine: -forward, 5'- GAC CCC TAA GGC TGA GGA AC-3', reverse, 5'- CTC TCC TGC CAT CTT TCG AG -3'-; IP-10: -forward, 5'ACC TCC AGT CTC AGC ACC ATG -3', reverse, 5'- TGG GAG GAT GGC AGT GGA AG-3'-; MCP-1: -forward, 5'- AAA CTG AAG CTC GCA CTC TCG C - $3^{\prime}$, reverse, $5^{\prime}$ - ATT CTT GGG TTG TTG AGT GAG T -3'-; RIG-I: -forward, 5'- GCA TAT TGA CTG GAC GTG GCA -3', reverse, 5'- CAG TCA TGG CTG CAG TTC TGT C -3'-; TLR3: -forward, 5'- CTC AGA AGA TTA CCA GCC GCC - $3^{\prime}$, reverse, 5'CCA TTA TGA GAC AGA TCT AAT G - - ${ }^{\prime}-$; and $18 \mathrm{~S}$ rRNA: -forward, 5'-ACT CAA CAC GGG AAA CCT CA-3', reverse, 5'-AAC CAG ACA AAT CGC TCC AC-3'- (Takara Bio Inc, Otsu, Japan). The relative mRNA abundance in the patients was calculated by the $2^{-\Delta \Delta C t}$ method. ${ }^{1-13}$

For a pilot immunofluorescence staining for fractalkine, the OCTembedded tissue specimens, stored in good condition, from six patients with IgAN and two patients with TBMD were cut into $5 \mu \mathrm{m}$-thick sections using a cryostat, briefly fixed in cold acetone and then air-dried; the slides were then washed in phosphate-buffered saline (PBS) solution immediately before the immunohistochemical procedure. The slides were incubated with anti-human rabbit fractalkine antibody (1:100, Santa Cruz Biotechnology, CA, USA), followed by incubation with fluorescein isothiocyanate (FITC)conjugated secondary antibodies (1:40) (Santa Cruz Biotechnology). The degree of intensity of immunostaining was determined as on a scale of $0-2(0$, negative or trace; 1 , positive; 2 , strongly positive). 
Data were expressed as the mean $\pm \mathrm{SD}$ and analyzed using the Mann-Whitney's $U$-test, Wilcoxon's $U$-test, and Spearman rank correlation coefficient, when appropriate, employing the computer software SPSS (SPSS, Chicago, IL, USA). The level of statistical significance was set at $P<0.05$.

\section{RESULTS}

The summary of clinical characteristics of study participants are shown in Table 1. The summary of urinary composition of the urinary sediments from patients is shown in Table 2 . The results showed that the expressions of CCL5, fractalkine and RIG-I were significantly higher in the urinary sediment of IgAN patients than in that of controls $(P<0.05$, respectively) while the expressions of IP-10 and TLR3 in the urinary sediment showed no significant differences between IgAN patients and controls. The expression of MCP-1 in the urinary sediment of IgAN patients showed more of a tendency to increase than in that of controls $(P=0.053)$ (Fig. 1). However, we found no correlation between the expressions of these functional molecules in the urinary sediment and

Table 1 Summary of clinical characteristics of the study participants

\begin{tabular}{lccl}
\hline Variable & $\operatorname{IgAN}(n=20)$ & Control $(n=4)$ & $P$ \\
\hline Age (years) & $8.5(7.0-14.3)$ & $14.0(11.8-15.3)$ & NS \\
Gender, $n$ (female/male) & $10 / 10$ & $1 / 3$ & $N^{a}$ \\
Creatinine (mg/dL) & $0.50(0.38-1.60)$ & $0.55(0.48-0.63)$ & NS \\
Occult blood in urine & $3(1-3)$ & $0.25(0-1.13)$ & NS \\
U-prot/cre & $1.16(0.12-1.97)$ & $0.09(0.08-0.13)$ & $<0.05$ \\
Activity index & $3(2-4)$ & 0 & $<0.01$ \\
Chronicity index & $3(2-3.25)$ & 0 & $<0.01$ \\
\hline
\end{tabular}

Data are presented as the median with the interquartile range (IQR; Q1 (lower quartile) and Q3 (upper quartile)) in parenthesis, unless otherwise indicated;

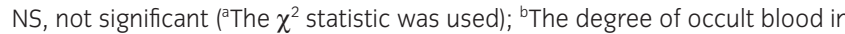
urine was determined as on a scale of $0-3(0$, none; 1 , approximately 20 red blood cells $(\mathrm{RBCS}) / \mu \mathrm{L} ; 2$, approximately $80 \mathrm{RBCs} / \mu \mathrm{L}$; 3, approximately 200 $\mathrm{RBC} / \mu \mathrm{L})$ the value of Up/cr in patients with IgAN. Also, there were no correlations between the other clinical parameters, such as degrees of occult blood in urine and serum levels of albumin, and the expressions of these molecules in the patients' urinary sediment. In this study, there were no significant correlations between the history of gross haematuria in IgAN patients and the urinary gene expressions of target molecules. As for the correlation between the histological indices (AI and CI) and the expressions of CCL5, fractalkine and RIG-I in urinary sediment of the patients, a significant correlation was observed between the expression of fractalkine and the AI $\left(r_{s}=0.510, P=0.022\right)$ and CI $\left(r_{s}=0.601\right.$, $P=0.005$ ) (Fig. 2), while no correlation was observed between the indices and the expressions of CCL5 and RIG-I. As for each parameter of histologic index, such as percentage of crescent formation, no correlation between each index and urinary gene expression of fractalkine was observed in this study. Interestingly, a significantly increased intensity of fractalkine immunostaining in the mesangial area and capillary loop was observed in the biopsy specimens of the patients with more than moderately mesangial proliferation. On the other hand, no significant fractalkine intensity of immunostaining was observed in the specimens of the patients with mild proliferative lesion or TBMD (Fig. 3). However, no significant correlation between the grade of fractalkine intensity and the level of urinary gene expression was observed in this pilot study, partly because of small sample size.

\section{DISCUSSION}

It has been reported that urine samples sometimes reflect a glomerular lesion more directly than blood samples, and are easily obtained in a non-invasive fashion. ${ }^{14}$ Although gene expression in urinary sediment is thought to be instability, ${ }^{16}$ the presence of mRNA implies local production, whereas determining protein levels may reflect both local production

Table 2 Summary of cellular composition of the urinary sediments from study patients

\begin{tabular}{lcc}
\hline Variable & IgAN $(n=20)$ & Control $(n=4)$ \\
\hline RBC (/HPF) & $47.15(14.95-100)$ & $7.70(0.58-36.1)$ \\
WBC (/HPF) & $5.60(1.53-12.75)$ & $0.65(0.50-0.93)$ \\
Squamous cell (/HPF) & $0.45(0.20-1.28)$ & $0.30(0.28-0.30)$ \\
Renal tubular epithelial cell (/HPF) & $1.25(0.23-3.43)$ & $0.10(0-0.08)$ \\
Urothelium (/HPF) & $0(0-0)$ & $0(0-0.03)$ \\
Red blood cell casts (/LPF) & $0(0-0.2)$ & NS \\
White blood cell casts (/LPF) & $0(0-0.1)$ & NS \\
Epithelial cell cast (/LPF) & $0.3(0.08-0.73)$ & NS \\
Fatty casts (/LPF) & $0.15(0-1.1)$ & $<0.05$ \\
Granular casts (/LPF) & $0(0-0.1)$ & NS \\
Hyaline casts (/LPF) & $0.9(0.4-1.25)$ & NS \\
Waxy casts (/LPF) & $0(0-0.2)$ & $<0.05$ \\
\hline
\end{tabular}

Data are presented as the median with the IQR in parenthesis, unless otherwise indicated. HPF, high power field; IgAN, immunoglobulin A nephropathy; LPF, low power field; NS, not significant; RBC, red blood cells; WBC, white blood cells. 


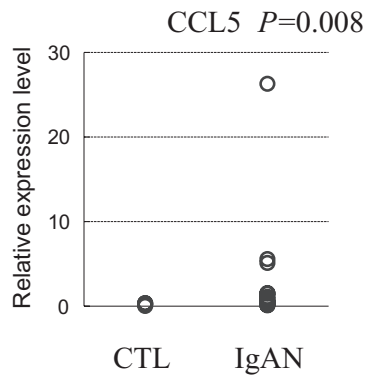

MCP-1 $P=0.053$

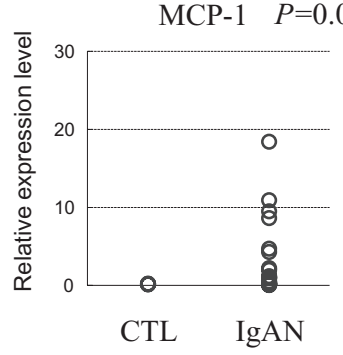

$r=0.510, P=0.022$
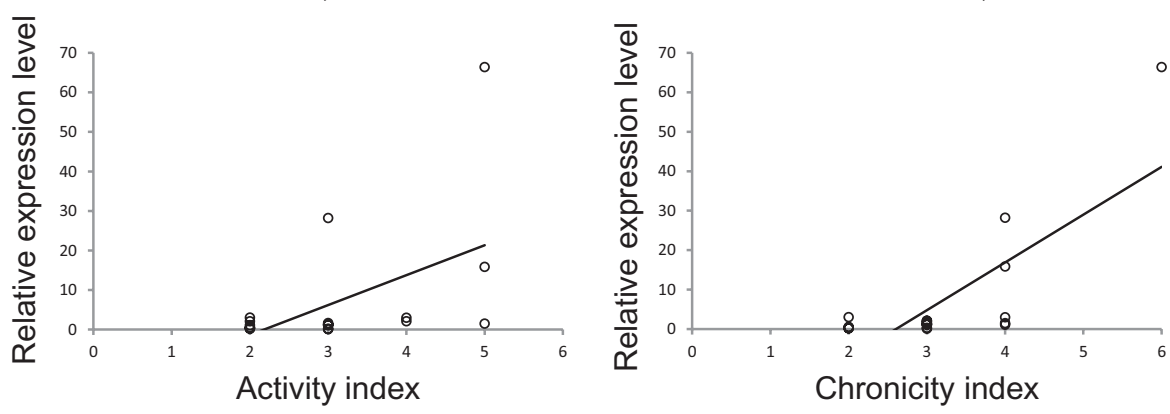

Fig. 1 Relative gene expressions in urinary sediment. Significant differences in the mRNA expressions of CCL5, fractalkine and RIG-I between IgAN patients and non-inflammatory controls were observed (Upper panel, from left to right show the results of CCL5, IP-10 and fractalkine, respectively. Lower panel, frome left to right show the results of MCP-1, RIG-I and TLR3, respectively). CTL, control; IgAN, IgA nephropathy; CCL5, CC chemokine ligand 5; IP-10, interferon- $\gamma$-induced protein 10; MCP-1, monocyte chemoattractant protein 1; RIG-I, retinoic acid-inducible gene-I; TLR3, Toll-like receptor 3 .
Fig. 2 Correlation of urinary mRNA expression of fractalkine and histologic indices. Urinary mRNA expression of fractalkine shows a significant association with the $\mathrm{Al}$ (left) and $\mathrm{Cl}$ (right), respectively. $\mathrm{Al}$, activity index; $\mathrm{Cl}$, chronicity index. and leakage from systemic circulation. ${ }^{14}$ Thus, measurement of mRNA expression in urinary sediment could be used as a non-invasive method for predicting histologic severity in IgAN in children. ${ }^{12,13}$ Our previous studies of children with newly diagnosed IgAN and PN showed Thl predominance in urinary sediment compared to controls, and that this Thl/ Th2 imbalance might be involved in the pathogenesis of regional inflammation. ${ }^{12,13}$ Given the implication of innate immunity in the pathogenesis of immunocomplex-mediated glomerulonephritis, ${ }^{1-7}$ we have examined the TLR3 signalling cascades in cultured human MCs and found pivotal roles of regional activation of Thl-related functional molecules: CCL5, fractalkine, IP-10, MCP-1, RIG-I and TLR3. ${ }^{1,4,5,8,9}$

Fractalkine/CX3CLl is known as a chemokine that induces the chemotaxis and activation of monocytes/ neutrophils expressing its receptor. To date, fractalkine expression in mesangial lesions has been reported to be significantly correlated with histopathological disease activity in a rat model of prolonged proliferative glomerulonephritis, ${ }^{17}$ and in human and mouse models of lupus nephritis (LN). ${ }^{18,19}$ Furthermore, it has recently been reported that upregulation of glomerular and urinary expression of fractalkine promotes the onset of gross haematuria in patients with IgAN. ${ }^{3,6}$ Thus, fractalkine is thought to be involved in the pathogenesis and aggravation of IgAN, PN and LN. ${ }^{1,6,8,16-18}$ However, the implications of urinary mRNA expression of fractalkine in children with IgAN remain to be determined. In this pilot study, we firstly found an increased mRNA expression of fractalkine in urinary sediment in IgAN, and that the expression reflected histologic severity, although our result remains preliminary. Previously, we also found a strong association between increased urinary fractalkine protein concentrations (measured by ELISA) and the severity of haematuria in children with IgAN. ${ }^{16}$ Based on recent studies regarding glomerular expression of fractalkine in patients with IgAN, ${ }^{1,3,6}$ fractalkine is reportedly thought to be a strong inducer of glomerular basement membrane (GBM) injury, resulting in massive haematuria. ${ }^{3,6}$ Thus, the urinary fractalkine protein concentration may be a predictor of inflammatory GBM damage in selected patients with IgAN, ${ }^{16}$ whereas the mRNA expression in urinary sediment may reflect histologic severity, although this theory remains to be further determined in future studies. In this study, we did not observe the relationship between the grade of occult blood in 

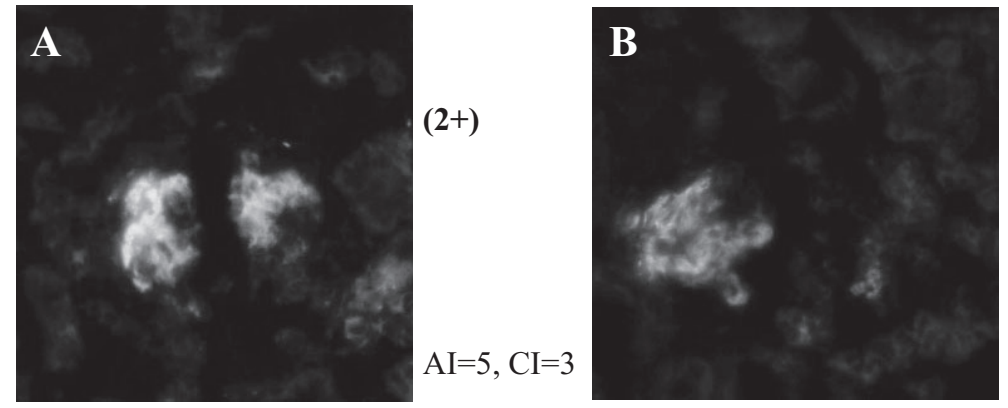

$(1+)$

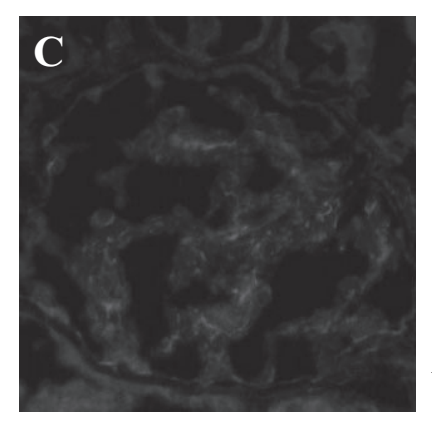

$\mathrm{AI}=2, \mathrm{CI}=2$
D

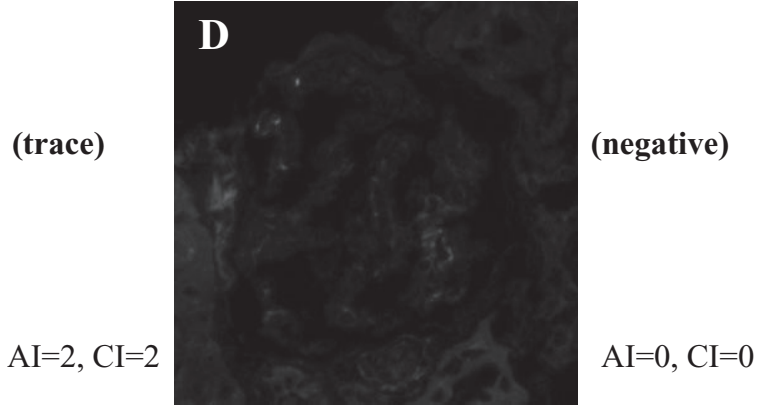

urine or the history of gross haematuria and urinary gene expression of fractalkine in IgAN patients. We now think that small sample size and timing of sample collection may be responsible for this issue. This issue remains to be elucidated.

As for the other mRNA expressions of functional molecules in urinary sediment, we observed no significant correlation between the expressions of CCL5, MCP-1 and RIG-I and histologic severity in our study participants. Recently, Chan et al. reported that mRNA expression of RANTES (CCL5) in urinary sediment was increased in patient with active LN. ${ }^{10}$ It has been reported that glomerular expression of MCP-1 is involved in the pathogenesis and subsequent tissue injury in paediatric-onset LN. ${ }^{20}$ Also, we observed an increased expression of RIG-I in urinary sediment in patients with LN. ${ }^{11}$ However, these results were obtained from LN patients. In this context, we recently found stronger activation of innate immunity in patients with LN than that of IgAN. ${ }^{21}$ Thus, we think this issue may have accounted for the difference in the mRNA expressions of urinary sediment.

We could not always confirm the cellular composition of the urinary sediment, although microscopic examination of urine samples from our patients showed that the sediments were composed mainly of erythrocytes, mononuclear leukocytes, and tubular epithelial cells (Table 2). Chan et al. previously reported that $\mathrm{T}$ lymphocytes were the major components of urinary mononuclear cells and tubular epithelial cells in active $\mathrm{LN} .{ }^{22}$ In an interesting experimental setting using poly IC, podocyte damage is reportedly seen through the signalling cascades via TLR3 activation. ${ }^{19}$ Wang and Szeto reported that glomerular inflammation-induced detached podocyte can be detected in urine sediment. ${ }^{14}$ Taken together, although it is difficult to determine the exact cellular origin of mRNA production in gene expression studies, ${ }^{14}$ a diversity of cell types associated with glomerular injury may be found in urine sediment. Therefore, despite limitations, we believe that measurement of mRNA expression of fractalkine would be a useful non-invasive approach for prediction of glomerular inflammation in children with IgAN.

In this pilot study, we first found the possible usefulness of measurement of urinary mRNA expression of fractalkine in children with IgAN. However, we did not examine that in healthy children in addition to patients with glomerular diseases, since it was not possible to obtain the samples during the study period. Thus, our present findings remain preliminary. Further detailed studies are needed to draw conclusions.

\section{ACKNOWLEDGEMENT}

This work is supported by a grant 2011 from Okinaka Memorial Institute for Medical Research (H. T.)

\section{REFERENCES}

1. Aizawa-Yashiro T, Imaizumi T, Tsuruga K et al. Glomerular expression of fractalkine is induced by polyinosinic-polycytidylic acid in human mesangial cells: Possible involvement of fractalkine after viral infection. Pediatr. Res. 2013; 73: 180-86.

2. Coppo R, Amore A, Peruzzi L, Vergano L, Camilla R. Innate immunity and IgA nephropathy. J. Nephrol. 2010; 23: 626-32.

3. Cox SN, Sallustio F, Serino G et al. Activated innate immunity and the involvement of CX3CRl-fractalkine in promoting hematuria in patients with IgA nephropathy. Kidney Int. 2012; 82: 548-60.

4. Imaizumi T, Aizawa-Yashiro T, Tsuruga $\mathrm{K}$ et al. Melanoma differentiation-associated gene 5 regulates the expression of a chemokine CXCL10 in human mesangial cells: Implications for 
chronic inflammatory renal diseases. Tohoku J. Exp. Med. 2012; 228: 17-26.

5. Imaizumi T, Aizawa-Yashiro T, Watanabe S et al. TLR4 signaling induces retinoic acid inducible gene-I and melanoma differentiation-associated gene 5 in mesangial cells. J. Nephrol. 2013; 26: 886-93.

6. Sugimoto K, Fujita S, Miyazawa T, Okada M, Takemura T. Periodic fever, aphthous adenitis, pharyngitis, and adenitis syndrome (PFAPA) and IgA nephropathy. Pediatr. Nephrol. 2013; 28: 151-4.

7. Yamashita M, Millward CA, Inoshita H et al. Antiviral innate immunity disturbs podocyte cell function. J. Innate Immun. 2013; 5: $231-41$.

8. Tanaka H, Imaizumi T. Inflammatory chemokine expression via toll-like receptor 3 signaling in normal human mesangial cells. Clin. Dev. Immunol. 2013; 2013: 984708.

9. Imaizumi $\mathrm{T}$, Tanaka H, Matsumiya $\mathrm{T}$ et al. Retinoic acid-inducible gene-I is induced by double-stranded RNA and regulates the expression of CC chemokine ligand (CCL) 5 in human mesangial cells. Nephrol. Dial. Transplant. 2010; 25: 3534-9.

10. Chan RWY, Lai FMM, Li EKM et al. Messenger RNA expression of RANTES in the urinary sediment of patients with lupus nephritis. Nephrology (Carlton) 2006; 11: 219-25.

11. Tsugawa K, Oki E, Suzuki K, Imaizumi T, Ito E, Tanaka H. Expression of mRNA for functional molecules in urinary sediment in glomerulonephritis. Pediatr. Nephrol. 2008; 23: 395-401.

12. Tsuruga K, Oki E, Aizawa-Yashiro T, Yoshida H, Imaizumi T, Tanaka H. Potential Th1/Th2 predominance in children with newly diagnosed IgA nephropathy. Acta Paediatr. 2010; 99: 1584-6.

13. Tsuruga K, Watanabe S, Oki E et al. Imbalance towards Thl pathway predominance in purpura nephritis with proteinuria. Pediatr. Nephrol. 2011; 26: 2253-8.
14. Wang G, Szeto CC. Quantification of gene expression in urinary sediment for the study of renal diseases. Nephrology (Carlton) 2007; 12: 494-9.

15. Andreoli SP, Bergstein JM. Treatment of severe IgA nephropathy in children. Pediatr. Nephrol. 1989; 3: 248-53.

16. Aizawa T, Imaizumi T, Tsuruga $\mathrm{K}$ et al. Urinary fractalkine and monocyte chemoattractant protein-1 as possible predicators of disease activity of childhood glomerulonephritis. Tohoku J. Exp. Med. 2013; 231: 265-70.

17. Ito Y, Kawachi H, Morioka Y et al. Fractalkine expression and the recruitment of $\mathrm{CX}_{3} \mathrm{CRI}^{+}$cells in the prolonged mesangial proliferative glomerulonephritis. Kidney Int. 2002; 61: 2044-57.

18. Nakatani K, Yoshimoto S, Iwano M et al. Fractalkine expression and $\mathrm{CD} 6^{+}$monocyte accumulation in glomerular lesions: Association with their severity and diversity in lupus models. Am. J. Physiol. Renal Physiol. 2010; 299: F207-16.

19. Yoshimoto S, Nakatani K, Iwano M et al. Elevated levels of fractalkine expression and accumulation of $\mathrm{CD} 6^{+}$monocytes in glomeruli of active lupus nephritis. Am. J. Kidney Dis. 2007; 50: 47-58.

20. Marks SD, Williams SJ, Tullus K, Sebire NJ. Glomerular expression of monocyte chemoattractant protein-1 is predictive of poor prognosis in paediatric lupus nephritis. Nephrol. Dial. Transplant. 2008; 23: 3521-6.

21. Watanabe S, Imaizumi T, Tsuruga K et al. Glomerular expression of myxovirus resistance protein $1(\mathrm{Mxl})$ in human mesangial cells: Possible activation of innate immunity in the pathogenesis of lupus nephritis. Nephrology (Carlton) 2013; 18: 833-7.

22. Chan RWY, Lai FMM, Li EKM et al. Urinary mononuclear cell and disease activity of systemic lupus erythematosus. Lupus 2006; 15: $262-7$. 